\title{
Cervical Ganglioneuroma in A Four Year Old Boy
}

\author{
Deepthi Chandrika Chintalapudi ${ }^{1}$, Savitri Mallikarjun Nerune ${ }^{1 *}$, Vijaya L Patil ${ }^{2}$, S U Arakeri ${ }^{1}$ \\ ${ }^{1}$ Department of Pathology, BLDEU( Deemed to be University ) Shri B M Patil Medical College, Hospital and \\ Research Centre, Vijayapura.INDIA \\ ${ }^{2}$ Department of Surgery, BLDEU ( Deemed to be University ) Shri B M Patil Medical College, Hospital and \\ Research Centre, Vijayapura.INDIA
}

\begin{abstract}
Ganglioneuroma is a rare benign neurogenic tumour arising from the sympathetic system. Ganglioneuromas are most commonly seen in posterior mediastinum, retroperitoneum, adrenal gland and rarely seen in cervical region.GNs tend to occur in older children at the age of 5 to 7 years. Here, we present a case of cervical ganglioneuroma in a 4year old male child who presented with slow growing painless lateral neck swelling since birth.
\end{abstract}

Keywords: Children, Neck Mass, Neurogenic Tumour

\section{Introduction}

Ganglioneuromas occurring in the head and neck region are rare. As reported in literature, only $1-5 \%$ of the affected patients present with mass lesions over the cervical area. Ganglioneuromas (GNs) are rare benign tumour that arises from the peripheral derivatives of sympathoblasts constituting neural crest origin of cells [1]. They are usually seen in children and young adults less than 20 years of age with a slight female predominance [2]. Preoperative investigations like imaging techniques and fine needle aspiration cytology may play a supportive role in diagnosis of GNs, but the histopathological study will give the definitive diagnosis [1].

\section{Case Report}

A 4-year old-boy, presented with the slow growing mass in the left lateral side of the neck since birth slowly increasing in size. Mass was $5 \times 4 \times 2.5 \mathrm{~cm}$ firm in consistency and tender. There was no history of fever, cough or respiratory symptoms. No relevant medical, family and personal history. With provisional diagnosis of tubercular lymphadenitis/lymphoma, patient was sent for fine needle aspiration cytology (FNAC) to render the diagnosis. FNAC was done from left lateral neck mass and aspirated scanty blood mixed material. Slides were stained with Giemsa, Pap and H\&E. Microscopy showed moderate cellularity comprised of ganglion like cells of varying sizes. Individual cells were round to polygonal having eccentric round to oval nucleus with vesicular chromatin with prominent nucleoli and abundant cytoplasm. These cells were arranged in small clusters \& scattered singly.
Many binucleate cells and occasional giant cells were also seen. At foci scant fibromyxoid stroma and foci of spindle cells were noted. Background was hemorrhagic. No evidence of lymphoglandular bodies. Special stains like Ziehl Neelsen stain and Auramine\&Rhodamine stainsfor acid fast bacilli were negative. So it was reported as benign soft tissue tumour suggestive of Rhabdomyoma. Radiological and hormonal investigations were not done. Complete excision of the mass was done and sent for histopathology examination.

Gross examination of the excised specimen showed well encapsulated, bosselated pale pink globular tissue mass measuring $5 \times 4 \times 2.5 \mathrm{~cm}$ [Fig.1]. Cut section was solid, firm homogenous with foci of whorled appearance [Fig.2]. Sections were given from the representative areas and slides were stained with H\&E stain. Microscopy showed well encapsulated tumour tissue comprised of spindle shaped cells and singly scattered ganglion cells in a fibrillary background. Ganglion cells were large polygonal with round to oval eccentrically placed vesicular nucleus having prominent nucleoli with abundant eosinophilic cytoplasm. Stroma was comprised of bundles of longitudinal and transversely oriented Schwann cell suggestive of neuromatous stroma. There was no evidence of immature elements, atypia, mitosis and necrosis.[Fig.3,4].On immunohistochemical study schwann cells/stroma and ganglion cells were positive for S-100 and negative for desmin. So diagnosis of ganglioneuroma was given [Fig.5].On follow up examination for 6 months patient was completely alright and no evidence of recurrence. 


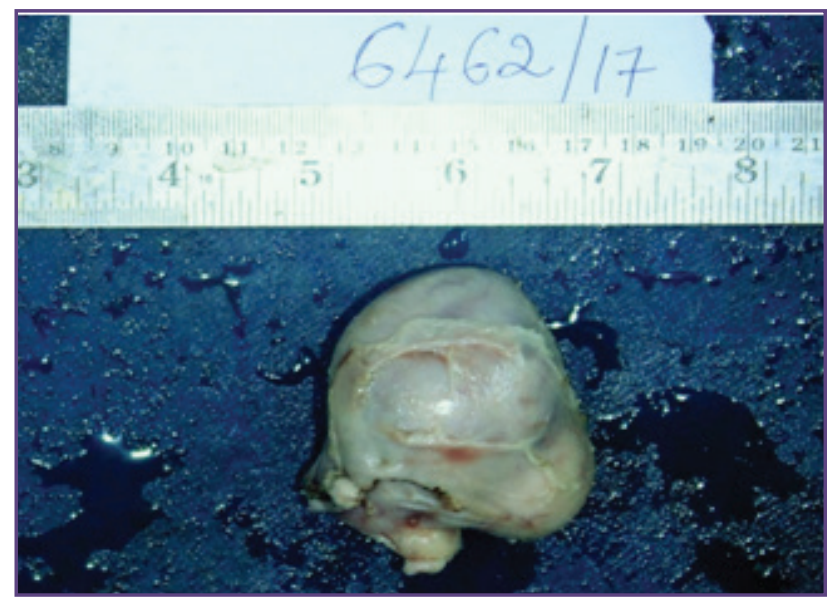

Fig. 1: Capsulated globular tissue mass measuring $5 \times 4 \times 2.5 \mathrm{~cm}$.

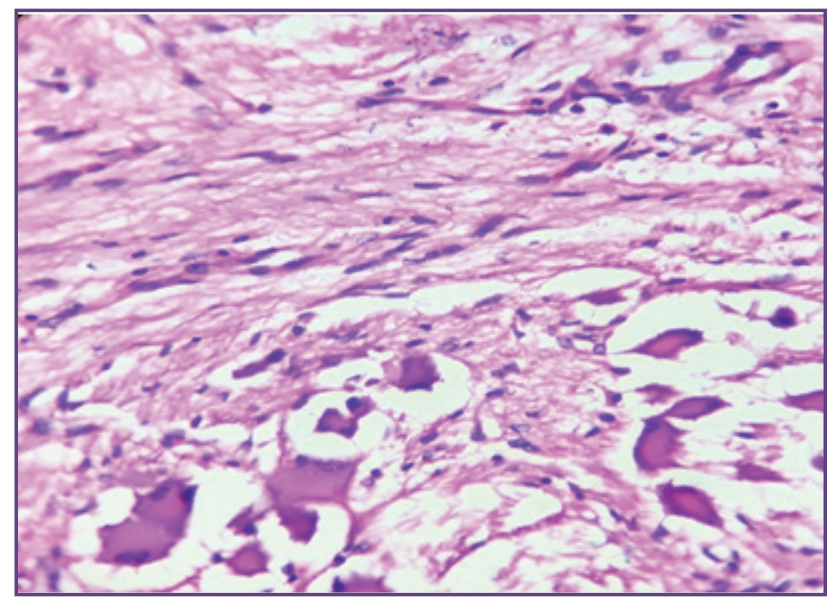

Fig. 3: Microphotograph showing neuromatous stroma suggestive of schwann cells. (H\&E 40x).

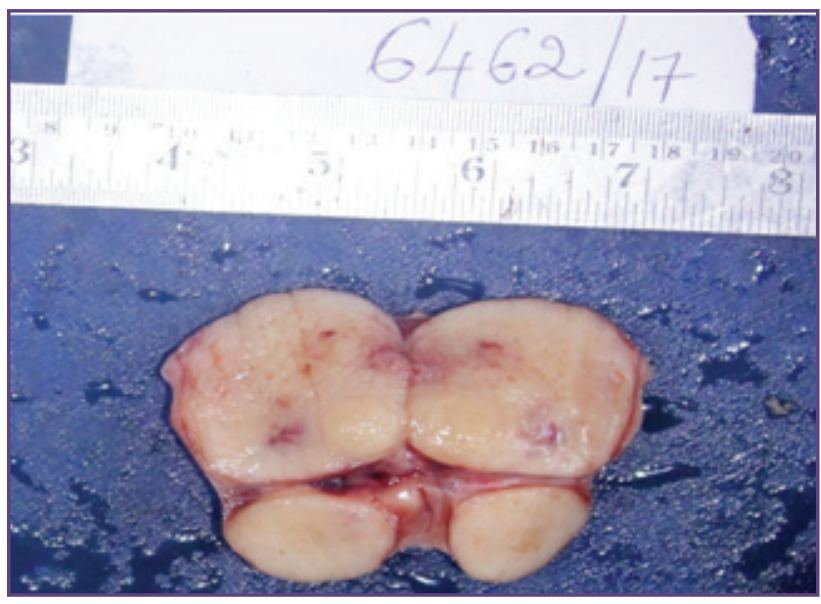

Fig. 2: Cut section showing solid, firm, homogenous and whorled appearance noted at places.

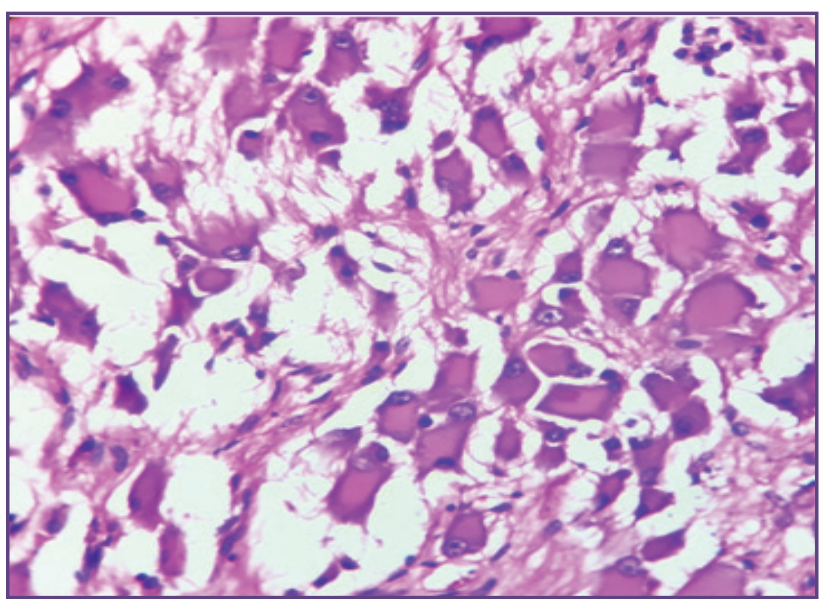

Fig. 4: Microphotograph showing large polygonal cells with eccentrically placed nucleus and prominent nucleoli suggestive of ganglion cells. (H\&E 40x).

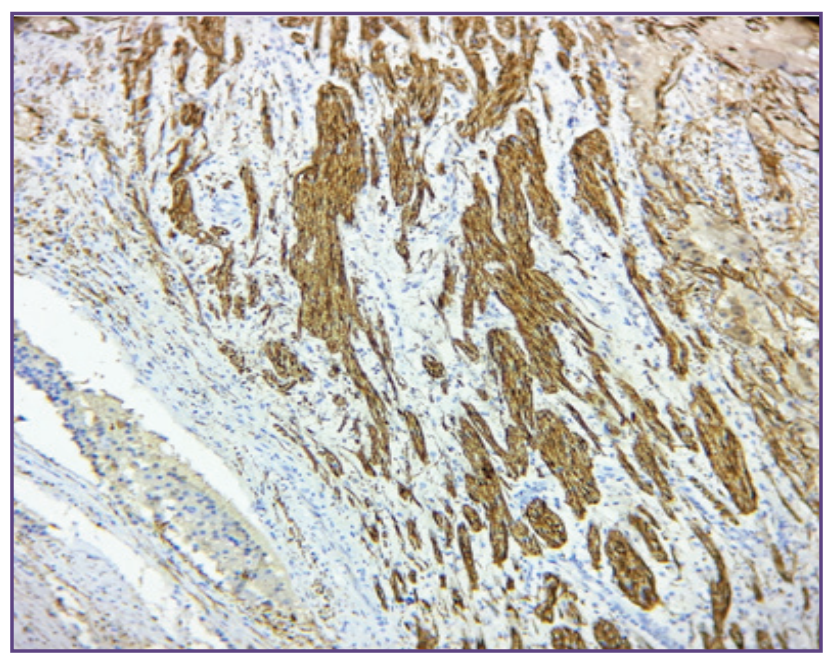

Fig. 5: IHC S-100 Positive (10x). 


\section{Discussion}

Loretz in 1870 first reported Ganglioneuroma and ganglioneuroma of neck was first reported by De Quervain in 1899[2].GN is a benign tumour arising from sympathetic ganglion, which frequently occurs at posterior mediastinum (41\%), retroperitoneum (37\%) and adrenal gland. Rarely, it may also present in the cervical region as a slow growing painless neck mass[1].Cervical ganglioneuromas are rare benign tumours comprising about $6 \%$ of childhood tumours[2]. GNs tend to occur in older children at the age of 5 to 7 years. As per pubmed literature search ganglioneuromas was reported with variable age distribution ranging from 4-47years with predominancein females[3,4,5,6]. However in the present case patient's age is 4 years, slightly younger than the age incidence reported in other studies.In the present case on cytology it was reported as benign soft tissue tumour suggestive of Rhabdomyoma, but only on histopathological examination\& IHC study diagnosis of ganglioneuroma was made.

GNs belongs to the family of neuroblastic tumours which encompass neuroblastoma and ganglioneuroblastoma[9]. The primordial tissue of sympathetic nervous system, is the seat of origin of neuroblastic neoplasm which is neural crest and neural tube derived. As per the Shimada classification the family of neuroblastic neoplasm includes, three histological patterns of the tumours based on maturation and differentiation of these neural crest cells andare categorised like: Neuroblastoma, Ganglioneuroblastoma and Ganglioneuroma[10].Neuroblastomas are undifferentiated neoplasms, whereas ganglioneuromas demonstrate evidence of schwannian stromal and ganglion cell differentiation[1].Neuroblastoma tend to be aggressive and occur in younger patients (average 2 years), whereas ganglioneuromas occur in older children (average 7 years). GNs may occur spontaneously or during the therapy for neuroblastomas with either chemotherapy or radiation therapy[7].In the present case no history of previous surgery or chemo/radiotherapy.

Ganglioneuromas, can present as a asymptomatic mass lesion or symptoms related to compression. There are also functional Ganglioneuromasthat release peptides such as Vasoactive Intestinal Peptides (VIP),Somatostatins and Neuropeptide Y (NPY). These may cause some symptoms like diarrhoea, sweating and hypertension[1].

In our patient, the mass was slow growing, painless and had no compressive symptoms or functional symptoms related to peptide secretion.

Grossly, in ganglioneuromas although the true capsule is infrequent they may appear to be encapsulated. In present case, it was encapsulated. Microscopically the Tumour tissue was comprised of spindle shaped cells arranged in interlacing bundles, loose myxoid stroma and ganglion cells. Ganglion cells are the most histologic characteristic feature of the ganglioneuroma[7].Indexed case also showed classic microscopic features of ganglioneuroma.

Differential diagnosis for GNs includes other neurogenic tumours like neurilemmoma, paraganglioma and neurofibroma.[8]. Clinically these tumours have similar clinical features as ganglioneuroma presenting as a solitary, nodular, slow growing painless mass with wide range of anatomical distribution. The microscopy of the neurilemmoma shows two different patterns and are usually recognised and designated as Antoni A and Antoni B areas which are comprised of spindle cells often arranged in a palisading fashion in an organoid arrangement suggestive of verocay bodies. Neurofibroma shows combined proliferation of all the elements of peripheral nerve: axons,schwann cells,fibroblasts and perineural cells. Schwann cells usually represent the predominant cellular element with elongated nuclei having wavy,serpentine configuration and pointed ends. Paraganglioma demonstrates well defined nests of cuboidal cells (Zell ballen) which are separated by highly vascularised fibrous septa. Individual tumour cells show abundant granular basophilic cytoplasm.

On immunohistochemical study schwann cells/stroma will be positive for S-100, synaptophysin, neurofilament protein (NF 1) and ganglion cells will be positive for S-100, synaptophysin, chromogranin A, neurofilament protein (NF 1), type IV collagen. The negative markers include Desmin, myogenin, EMA, cyokeratin, HMB45, CD99, CD45. In our case the tumour cells were positive for $\mathrm{S}-100$, and negative for desmin. Immunohistochemistry for neurilemmoma and paraganglioma show strong positivity for $\mathrm{S}-100$ where as it is $30 \%$ to $50 \%$ positive in neurofibromas. Presence of immature tissue elements, atypia, mitosis and necrosis excludes the diagnosis of ganglioneuroma.[8].In the present case also significant atypia, mitosis and necrosis was not seen. Hence it was concluded as ganglioneuroma.

Current advanced imaging techniques may be useful for evaluating the extent of the tumour and differential diagnosis preoperatively[7]. Imaging modalities like ultrasound may show homogeneous, hypoechoic, well circumscribed mass[1]. Computerized tomography (CT) most commonly reveals a well-encapsulated tumour with low attenuation and possible central densities. Magnetic Resonance Imaging (MRI) demonstrates intermediate signal intensity and non homogeneous contrast enhancement[7]. CT and 
MRI are more valuable imaging techniques in the diagnosis of ganglioneuroma. Although ganglioneuroma tends to be a more homogeneous tumour than neuroblastoma or ganglioneuroblastoma, it is not possible at imaging studies to discriminate among these three tumours [2].In the present case radiological investigations were not done as clinically it was not suspected as ganglioneuroma or ganglioneuroblastoma.

Surgical excision is the treatment of choice for cervical ganglioneuroma not only to confirm the diagnosis but also to prevent further growth and compression of adjacent structures[2]. No additional adjuvant therapy is necessary[1].These tumours are not aggressive to regrow or cause any other symptoms leading to any kind of morbidity and rarely may recur[2].

In our case complete excision of the tumour was done. Patient was completely alright after surgery. Follow up examination after 6 months revealed no evidence of recurrence.

\section{Conclusion}

Ganglioneuroma of the cervical region is a rare benign soft tissue neoplasm of neuroblastic origin of neural crest cells. Although average age for ganglioneuroma is 7 years it should be considered in the differential diagnosis of neuroblastic tumour, as neuroblastoma and ganglioneuroblastoma are having aggressive behaviour as compared to ganglioneuroma. Extensive study of the excised tissue should be done to rule out presence of immature element, necrosis, atypia before concluding the diagnosis as ganglioneuromaIn the present case also it was reported as rhabdomyoma on FNAC, and only on histopathology and immunohistochemical studies final diagnosis of ganglioneuroma was given.

\section{References}

1. Kiflu W, Nigussie T. Ganglioneuroma presenting as a neck mass: A case report.Ethiop Med J. 2017;55(1):69-71.

2. Dutta HK. Cervical Ganglioneuroma in a Child. SM J Pediatr Surg. 2016; 2(2): 1013.

3. MA J, Liang L, Liu H. Multiple cervical ganglioneuroma: A case report and review of the literature. 2012. Oncology letters 4:509-512.

4. Gary C, Robertson H, Ruiz B, Zuzukin V, Walvekar R.R. Retropharyngeal Ganglioneuroma Presenting with Neck Stiffness: Report of a Case and Review of Literature. Skull base. 2010.20 (5). Pg 371-74.

5. Gupta PK, Batish VK+, Deshpande GU, KhannaSk, Singh A.Rare presentation of a case of ganglioneuroma (a case report) mjafi 1997; $53: 82-84$.

6. Lee D,Choe WJ1,Lim SD. Ganglioneuroma of the Sacrum. Korean J Spine;2017, Volume 14 (3):106-108.

7. Albuquerque B S,Farias T P, Dias F L, TormanD.Surgical management of parapharyngeal ganglioneuroma: A case report and review of literature.ORL2013;75:240-244.

8. Ramani M, Krishna O.H.R, Reddy K.R, Chanakya, Sowjanya R. An interesting case of differentiated neuroblastoma - Ganglioneuroma of the neck in a 5 year old female child. Journal of evolution of medical and dental sciences.2013;2(24):4298-4301.

9. Shetty PK, KB, GnanaPrakash S, Shetty PK. Ganglioneuroma Always A Histopathological Diagnosis. Online J Health Allied Scs. 2010;9(4):19

10. Gopal A, Singh S, Srishar M, Khurana N. Cervical sympathetic chain ganglioneuroma: case report and review of literature. IOSR Journal Of Pharmacy.2015;vol 5(5):p 08-11.

*Corresponding author:

Dr. Savitri M.Nerune, AssociateProfessor, Department of Pathology. BLDEU's Shri B.M.Patil medical college, hospital and research centre, Vijayapura-586103INDIA

Phone: +9109008179806

Email: savitri.nerune@bldedu.ac.in

Financial or other Competing Interests: None. 